\title{
Lessons Learnt from Recent Trials in Ischemic Heart Disease
}

\author{
John M. Mandrola ${ }^{1}$ Sanjay Kaul ${ }^{2}$ Andrew Foy ${ }^{3}$ \\ ${ }^{1}$ Department of Cardiology, Baptist Health Louisville, Louisville, \\ Kentucky, United States \\ 2 Department of Cardiology, Cedars-Sinai Medical Center, Los \\ Angeles, California, United States \\ ${ }^{3}$ Department of Cardiology, Penn State College of Medicine, Hershey, \\ Pennsylvania, United States
}

Thromb Haemost 2021;121:8-14.
Abstract
Keywords
- ischemic heart disease
- percutaneous coronary intervention
- coronary artery bypass graft
- placebo

\begin{abstract}
Address for correspondence John M. Mandrola, MD, Department of Cardiology, Baptist Health Louisville, 3900 Kresge Way, Louisville, KY 40207, United States (e-mail: john.mandrola@gmail.com).
\end{abstract}

\section{Recent Trials in Ischemic Heart Disease}

Numerous randomized controlled trials (RCTs) published over the past 4 years have informed the care of patients with stable ischemic heart disease (SIHD). The purpose of this clinical focus article is to offer a summary and critical appraisal of these trials.

\section{ISCHEMIA Trial}

Landscape Before and Key Design Features of ISCHEMIA The ISCHEMIA trial was conducted to resolve the equipoise regarding the utility of upfront invasive coronary angiography (ICA) and the efficacy of early revascularization plus guideline-directed medical therapy (GDMT) versus GDMT alone in high-risk patients with stable angina and moderateto-severe myocardial ischemia on stress imaging. The rationale to perform the trial was largely twofold. ${ }^{1}$ First, previous clinical trials ${ }^{2,3}$ in patients with SIHD, which found no reduction of myocardial infarction (MI) or death with upfront ICA and percutaneous coronary intervention (PCI) plus
GDMT, randomized patients after ICA. Knowledge of the anatomy likely led to a selection bias wherein high-risk patients were excluded from enrollment. Consider that in the COURAGE trial, $39 \%$ of enrolled patients had no to low amounts of ischemia on nuclear stress imaging. ${ }^{4}$ The second rationale for ISCHEMIA was the question of routine coronary angiography and revascularization after documented ischemia on a stress exam. Given the cost and potential complications of early invasive management, the ISCHEMIA authors felt it necessary from a public health and individual patient perspective to test this standard practice.

To determine the degree of ischemia that warranted inclusion into the trial, ISCHEMIA authors relied on an observational study conducted in the 1990s that reported that patients with an ischemic burden of $10 \%$ or greater had improved survival with early revascularization compared with medical therapy. ${ }^{5}$ As with nonrandomized observational studies, potential confounding is likely. Inclusion criteria for ISCHEMIA required at least moderate degrees of ischemia, defined by $\geq 10 \%$ on nuclear perfusion and $\geq 3 / 16$ segments on stress echocardiography. The original trial protocol required stress imaging, received

February 26, 2020

accepted after revision

July 15,2020 (c) 2021. Thieme. All rights reserved. Georg Thieme Verlag KG,

Rüdigerstraße 14,

70469 Stuttgart, Germany
DOI https://doi.org/ 10.1055/s-0040-1715830. ISSN 0340-6245. 
but trialists later amended the protocol to include patients with moderate to severe ischemia documented on exercise studies alone. This decision and the choice of ischemic burden is a matter of controversy. ${ }^{6}$

Two other unique features of ISCHEMIA deserve mention. The decision to randomize patients before angiography allowed for a comparison of two strategies: an early invasive (angiography with or without revascularization plus medical therapy) versus an early conservative (medical therapy alone) approach. Another unique design feature was use of blinded coronary computed tomography angiography (CCTA) before randomization. This had two effects: (1) by excluding patients without obstructive disease, it enriched the trial population with patients who had significant coronary artery disease (CAD), and (2) by excluding patients with left main disease, it ensured safety for referral of patients before the anatomy was known.

\section{ISCHEMIA Results}

The primary endpoint in ISCHEMIA was a composite of cardiovascular death, nonfatal MI, hospitalization for unstable angina, hospitalization for heart failure, or resuscitated cardiac arrest. A key secondary endpoint was death or nonfatal MI. Other secondary aims of the trial were to assess angina symptoms and quality of life.

The ISCHEMIA trial randomized 2,588 patients to the early invasive arm and 2,591 patients to the early conservative arm. $^{7}$ The median age of participants was 64 years and $77 \%$ were men. Nearly $80 \%$ of patients who had CCTA had multivessel disease, including $45 \%$ with $\geq 3$-vessel disease. After a median follow-up of 3.2 years, the adjusted hazard ratio (HR) for the primary endpoint was 0.93 (95\% confidence interval [CI] 0.80-1.08), which did not reach statistical significance. Cardiovascular death or MI did not differ significantly. Rates of all-cause death were nearly identical. There was no heterogeneity of treatment effect based on any baseline characteristic. For example, patients with triple vessel disease had more than double the rate of primary outcome events compared with patients with single vessel disease, but there was no statistically significant difference in the treatment outcome between the two arms. The crossover rate from the conservative arm to the invasive arm was $28 \%$ with $21 \%$ undergoing revascularization compared with $79 \%$ in the invasive arm. The yearly crossover rate in ISCHEMIA was $6.6 \%$, similar to the rate in COURAGE $(7.2 \%) .^{3}$

ISCHEMIA authors reported a significant improvement in angina control and quality of life with the invasive strategy in patients who had daily to weekly or at least monthly angina. ${ }^{8}$ However, the probability of a clinically important (defined by a Seattle Angina Questionnaire [SAQ] summary score of $>5$ ) improvement in SAQ summary score was high only in those patients with daily to weekly angina, which comprised just $21 \%$ of the cohort. In those with at least monthly angina (44\%), the probability of a clinically important improvement in SAQ summary score was low; and in those with no angina (35.4\%), the probability of a clinically important improvement in SAQ summary score was zero.
A key challenge to the interpretation of the angina relief observed in ISCHEMIA is the possibility of a placebo effect due to the open-label nature of the trial and lack of a placebo (sham) control. We will explore the placebo effect of revascularization further in the ORBITA trial discussion.

In a separately reported trial of patients with stable CAD, advanced chronic kidney disease (CKD), and moderate or severe ischemia (ISCHEMIA-CKD), an initial invasive strategy again did not significantly reduce death or nonfatal MI compared with a conservative approach. ${ }^{9}$ Prior trials in this space had generally excluded patients with advanced CKD and were subject to the criticism that they enrolled patients at lower baseline risk, and hence, less likely to experience benefit from an early invasive approach. Patients enrolled in ISCHEMIA-CKD, however, represented a very high-risk group of individuals. Compared with the main ISCHEMIA trial, patients in the conservative strategy arm in the CKD trial were nearly sevenfold more likely to die (27.8\% vs. $4.3 \%$ ) and nearly twice as likely to experience an MI (15.9\% vs. $8.5 \%$ ) over 3 years of follow-up. Unlike the main ISCHEMIA trial, no treatment benefit with PCI + GDMT was observed on angina relief in the overall trial population or even in patients with daily to weekly angina. ${ }^{9}$

The null results from ISCHEMIA-CKD highlight the complex interplay between multimorbidity and treatment effects from medical interventions. In addition to advanced CKD, patients in the ISCHEMIA-CKD trial had significantly higher rates of diabetes as well as peripheral vascular disease and stroke compared with the main ISCHEMIA trial. The hypothesis that treatment effect of PCI + GDMT would be amplified in high-risk patients was not validated.

In fact, the invasive strategy was associated with a significantly higher incidence of stroke (HR 3.76, 95\% CI 1.52-9.32) and death or initiation of dialysis (HR 1.48, 95\% CI 1.04-2.11).

\section{ISCHEMIA Controversies}

\section{Were the Results Predictable because Ischemic Burden was too Low?}

One might posit that the choice to set the entry criterion for ischemia at $10 \%$ could have been too low. In the observational study used to determine the $10 \%$ ischemic threshold, the difference in survival between revascularization and medical therapy only reached statistical significance at $20 \%$ or higher degree of ischemia. ${ }^{5}$ However, the results from both ISCHEMIA and previous trials of SIHD do not support this notion.

First, subgroup analyses from the main ISCHEMIA trial and ISCHEMIA-CKD trial failed to show significant heterogeneity of treatment effect for the severe ischemia subgroup compared with patients with lesser degree of ischemia in either trial. Second, in a substudy of COURAGE, Shaw et al studied 1,381 patients (60\% of total) who had baseline nuclear perfusion imaging. They found that the primary endpoint, death or MI, was not statistically different with either GDMT or GDMT + PCI in patients with no to mild (18 and $19 \%, p=0.92$ ) and moderate to severe ischemia (19 and $22 \%, p=0.53$, interaction $p$-value $=0.65) .{ }^{4}$ Third, Mancini 
et al studied a subset of 621 patients from COURAGE who had both baseline quantitative nuclear single-photon emission $\mathrm{CT}$ and quantitative coronary angiography. Anatomic burden and left ventricular ejection fraction, but not ischemic burden, was an independent predictor of outcomes. Neither anatomic burden nor ischemic burden (either alone or in combination) identified a patient profile benefiting preferentially from an invasive therapeutic strategy. ${ }^{10}$

\section{How to Incorporate CCTA in Clinical Practice?}

The use of blinded CCTA before randomization was mandated by trial protocol to ensure patients with nonobstructive CAD were not enrolled ( $\sim 14 \%$ of those screened) and to enhance safety by excluding patients ( $\sim 5 \%$ screened) with left main CAD (LMCAD). While the internal validity of the trial might have been improved by protocol-mandated blinded CCTA, its external generalizability remains challenging. CCTA is not currently considered standard of care prior to choice of treatment for patients with SIHD. Some would argue that based on the results of ISCHEMIA, CCTA should become standard of care in all patients with angina and moderate to severe ischemia for decision making regarding initial revascularization plus GDMT versus GDMT alone. This is reasonable, because, although left main disease is uncommon, the possibility of missing a serious lesion looms large. Conversely, making an incorrect diagnosis (obstructive CAD when the ischemia is attributable to nonobstructive disease) and initiating an incorrect treatment plan (revascularization instead of GDMT) is not inconsequential. A purist would argue that because ISCHEMIA was not designed to address CCTA versus no CCTA, and nearly one in four enrolled patients did not have a CCTA, additional trials would be needed to confirm or refute the necessity of defining the coronary anatomy following stress testing prior to implementation of either an initial invasive or conservative approach.

\section{ORBITA Trial}

In the ISCHEMIA trial, as in others, revascularization added to GDMT versus GDMT alone reduced the burden of angina. But these trials were unblinded and did not include a placebo (sham) control, raising the possibility of a placebo effect. For instance, simply being told that one has no significant disease has been shown to reduce chest pain (the so-called "faith healing" effect). And it is easy to imagine how the opposite would occur for an individual who is told of a significant blockage that is left "unfixed" (the so-called "subtraction anxiety" effect). ${ }^{11}$

ORBITA randomized patients $(N=200)$ with ischemic symptoms and single vessel disease to either a PCI procedure or a sham procedure on the background of GDMT. ${ }^{12}$ The primary endpoint of this 6-week trial was the difference in exercise time before and after the procedure. ORBITA was powered to detect an effect size of 30 seconds despite the fact that a previous unblinded study (ACME) found that angioplasty without stenting yielded a 96-second gain in exercise time over medical therapy. ${ }^{13}$ Furthermore, placebo-controlled trials have shown antianginal medical therapies provide improvements in exercise time of 48 to 55 seconds. ${ }^{13,14}$

In ORBITA, there was no significant difference in the primary endpoint: placebo-controlled difference in exercise time increment was 16.6 seconds ( $95 \% \mathrm{CI}-8.9$ to 42.0 , $p=0.200$ ). After adjustment for baseline differences with analysis of covariance, the mean difference improved to 21.4 seconds but remained nonsignificant $(95 \% \mathrm{CI}-3.4$ to $41.1, p=0.09) .{ }^{15}$ In a post hoc analysis, the investigators found that patient-reported freedom from angina assessed by SAQ was significantly higher in the $\mathrm{PCI}$ arm (odds ratio $2.47[1.30,4.72], p=0.006){ }^{16}$ And baseline ischemia assessed by dobutamine stress echocardiography was the only outcome that predicted placebo-controlled impact of $\mathrm{PCI}$ on anginal frequency ( $p$ interaction $=0.031$, unadjusted for multiplicity). However, the authors found no significant interactions between stress echocardiography score and any other patient-reported response variables such as freedom from angina, physical limitations, and quality of life. ${ }^{17}$

There are several potential reasons why patients with angina did not improve with PCI in the main ORBITA results. First, the trial may have been underpowered: the standard deviation (SD) of 75 seconds used for sample size estimation was smaller than the SD of 132 seconds observed in ACME. The latter SD would have required a sample size of 608 . Second, the primary endpoint might not have been sensitive to the intervention in patients with mild symptoms. In ORBITA, $24 \%$ of patients had no or minimal angina. This is consistent with ISCHEMIA wherein $35 \%$ of patients with no or minimal angina at baseline did not derive any symptomatic benefit with revascularization. Third, ORBITA limited enrollment to patients with single-vessel CAD.

ORBITA informs the interpretation of health-related outcomes in ISCHEMIA as it strongly suggests the possibility that improvement in anginal symptoms with revascularization might in part be related to a placebo effect. Caveats include the fact that ORBITA enrolled patients with ischemic symptoms and single vessel disease with follow-up limited to 6 weeks, while ISCHEMIA enrolled patients with anginal symptoms, demonstrable ischemia, and $\geq 1$ vessel CAD followed for a median of 3.2 years. Stratifying health-related outcomes according to number of vessels involved $(1,2$, or 3 or more vessel CAD) and duration of follow-up (3 months, 12 months, and 3.2 years) in ISCHEMIA would be informative in this regard. We also eagerly await the results of ORBITA-2 which will test the placebo effect of $\mathrm{PCI}$ in a larger cohort $(n=400)$ of patients with documented severe CAD who are not on background anginal therapy and may include patients with more than one vessel disease. ${ }^{18}$

\section{Impact of ISCHEMIA (and ORBITA) on the Clinical Management of Patients with SIHD}

The results of ISCHEMIA are consistent with previous trials that found no convincing evidence of mortality or MI reduction with revascularization. ISCHEMIA results reinforce the recommendations endorsed by the SIHD guidelines issued by the American College of Cardiology/American Heart Association in 
$2012,{ }^{19}$ that is, revascularization should only be considered for limiting angina with severe or extensive myocardial ischemia, hemodynamically significant stenosis, and insufficient response to GDMT. Based on the totality of the evidence, SIHD patients without high-risk features (such as class IV angina, high-risk stress test, or high-risk anatomy such as LMCAD) should be started on an initial strategy of therapeutic lifestyle (TLS) intervention aimed at risk factor control plus GDMT. Revascularization can be reserved for a later time in the minority of patients whose symptoms or quality of life are either refractory or unacceptable on medical therapy with little risk that an unfavorable event including sudden cardiac death (1.3\% in ISCHEMIA) will intervene. Crucially, over twothirds to three-fourths of patients will not require revascularization over a 5- to 7-year follow-up with this strategy. For patients with high-risk features and estimated glomerular filtration rate $>30 \mathrm{~mL} / \mathrm{min} / 1.7 \mathrm{~m}^{2}$, a strategy of prompt revascularization plus GDMT and TLS might be reasonable with the choice between coronary artery bypass graft (CABG) versus $\mathrm{PCI}$ driven by the complexity of CAD, presence of diabetes, and left ventricular systolic dysfunction. Incorporating the patient's value judgments and preferences in shared decision-making is appropriate. For patients unwilling or unable to take GDMT long term, an initial strategy of revascularization may be reasonable for symptom relief, as long as they are duly informed that it will not make them live longer, only feel better.

\section{EXCEL and NOBLE Trials of Left Main Coronary Artery Disease}

Two large trials, EXCEL ${ }^{20,21}$ and NOBLE, ${ }^{22}$ have recently published results comparing PCI with drug-eluting stents (DESs) versus CABG in patients with LMCAD and low to medium anatomical complexity. Both EXCEL and NOBLE were designed as noninferiority (NI) trials.

EXCEL randomized 948 patients to PCI with DESs and 957 to CABG. The primary outcome was a composite of major adverse cardiac events (MACE) including death, nonfatal stroke, or nonfatal MI, which included periprocedural MI (PPMI). NOBLE randomized 598 patients to PCI with DES and 603 patients to CABG. The primary outcome of NOBLE was a composite of all-cause mortality, nonprocedural nonfatal MI, repeat revascularization, or nonfatal stroke (major adverse cardiac and cerebrovascular events).

The differences in primary endpoints deserve mention. For the definition of MI, EXCEL authors chose to include PPMI as defined by the Society for Cardiovascular Angiography and Interventions (SCAI) criterion for MI, with the Third Universal Definition of Myocardial Infarction (UDMI) criterion prespecified as a secondary outcome. ${ }^{23}$ The SCAI definition uses the same biomarker threshold for PCI and CABG to minimize ascertainment bias and allows for biomarker only defined cases; the UDMI, on the other hand, requires additional proof of MI (such as clinical signs and electrocardiogram changes) and uses variable biomarker thresholds, higher for CABG than PCI $(10 \times$ vs. $5 \times$ the 99 th percentile upper reference limit). Thus, one could argue the SCAI definition is biased against CABG because of greater biomarker elevation with the more invasive procedure. Nearly 85\% of PPMIs in EXCEL were defined on the basis of biomarker elevation criterion alone. NOBLE investigators included only nonprocedure-related MI which avoids this controversy entirely.

The second difference in primary endpoint was that NOBLE included repeat revascularizations in the composite whereas EXCEL did not. The repeat revascularization endpoint is controversial; some argue that it is biased against PCI as it includes revascularization due to progression of disease in segments remote from the left main site which are bypassed with CABG. Repeat revascularization is also a "softer" endpoint, as it is decided by individual and geographic practice preference. Others, however, feel that repeat revascularization in a left main trial is a reasonable endpoint, as it involves CABG in $25-30 \%$ of cases. ${ }^{24}$

A third difference in the two trials was the NI margin of the primary endpoints. In EXCEL, NI was set at a $4.2 \%$ risk difference at 3-year follow-up, while a risk ratio margin of 1.35 was used in NOBLE. Due to slower than expected event accrual, NOBLE follow-up was extended from 2 to 5 years. Five-year follow-up results of EXCEL were planned; however, it was not prespecified whether the trial would be analyzed as a NI or a superiority trial.

- Table 1 compares the divergent results of the two trials. In NOBLE, all-cause death at 5 years was not significantly different (8.7\% in CABG vs. 9.4\% in PCI, HR 1.08, 95\% CI 0.7-1.6), whereas in EXCEL, 5-year all-cause death was 3.1\% lower in the CABG arm (9.9\% vs. $13.0 \%$, HR $1.38,95 \%$ CI 1.03-1.85). Of note, CABG-related all-cause death rate was numerically similar in the two trials $(8.7 \% \mathrm{~N}$ vs. $9.4 \% \mathrm{E})$; however, PCI-related allcause death rate was higher in EXCEL (9.4\% N vs. 13\% E). This might be related to the fact that EXCEL enrolled more patients with a higher SYNTAX score (> 32), an exclusion criterion compared with NOBLE. SYNTAX score is a predictor of poor outcomes with PCI but not CABG.

Differing components of the primary composite endpoints in the two trials makes interpretation of the primary endpoint challenging. In NOBLE, superiority of CABG was driven by significantly lower rates of MIs and repeat revascularizations. In EXCEL, NI was met at 3 years despite a $2.3 \%$ higher rate of allcause death in the PCI arm because the latter was associated with a $2.2 \%$ reduction in MI, mostly driven by PPMI ( $60 \%$ of total MIs). In both trials, stroke rates were low $(<5 \%)$ and not statistically different in the two arms.

The main driver of better outcomes for PCI early on in EXCEL was the higher rates of PPMI in the CABG arm. This is a matter of debate because heart surgery will cause greater release of cardiac enzymes than PCI, thereby amplifying the PPMI events after surgery. How one defines MI matters. A Korean observational study of approximately 7,600 patients who had either PCI or CABG showed widely disparate rates of MI depending on the definition used. ${ }^{25}$ These ranged from $19 \%$ for PCI versus $3 \%$ for CABG using the Second UDMI, to $5.5 \%$ for PCI versus $18.3 \%$ for CABG for the SCAI definition. Further evidence that the MI definition in EXCEL was biased against CABG comes from a comparison of MI rates in the surgery arms of EXCEL versus SYNTAX, ${ }^{26}$ an RCT comparing $\mathrm{PCI}$ versus CABG in patients with multivessel or left main 


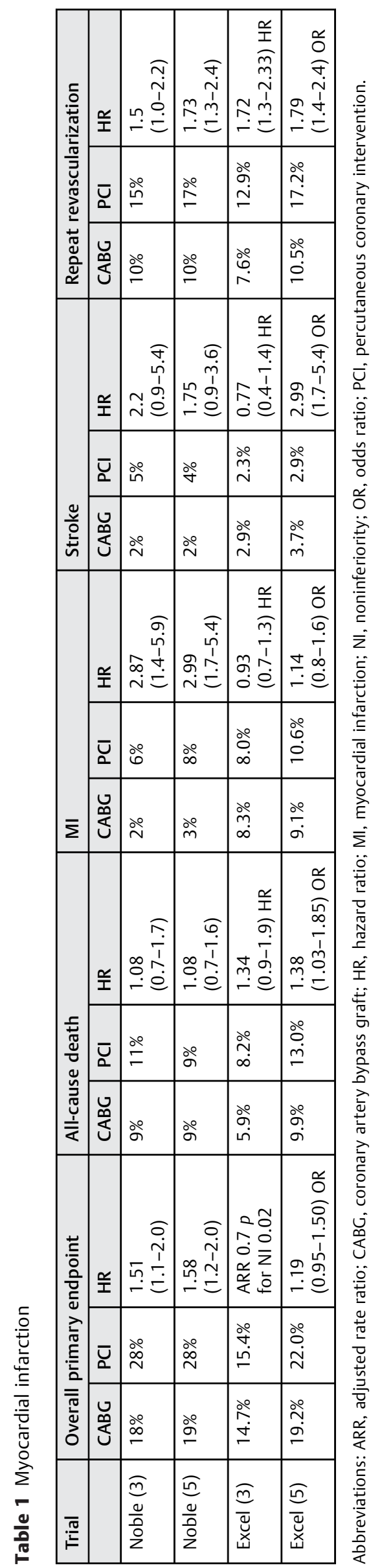

disease. In EXCEL, the MI rate in the CABG arm at 30 days was 6.2\%. In SYNTAX, the MI rate in the CABG arm at 1 year was only $3.3 \%$. This difference is likely due to the fact that in SYNTAX, the MI definition required new $\mathrm{Q}$ waves in addition to biomarker elevation criteria.

\section{EXCEL Controversy}

The 5-year results of EXCEL were controversial for three reasons. First, the rate of all-cause death was 3.1\% higher with $\mathrm{PCI}$ at 5 years, yet the investigators concluded that the two procedures were comparable because the primary MACE endpoint was not statistically different (22\% PCI vs. $19.2 \%$ CABG; $2.8 \%, 95 \% \mathrm{CI}-0.9 \%, 6.5 \%, p=0.13)$. This implies that the investigators analyzed the 5-year data as a superiority trial where the null hypothesis of no difference was not rejected. In fact, the trial had a planned NI assessment at 3 years. Had the investigators used the same NI analysis at 5 years, they would have failed to establish NI as the upper bound of the difference of $6.5 \%$ would exceed the NI margin of $4.2 \%$. It is misleading to interpret a trial result as "not significantly different" or "comparable" when a trial designed as a NI trial would not even have met NI.

Second, the MI rates determined by the Third UDMI (a secondary outcome) were not reported either in the 3year or the 5-year results of EXCEL. Shortly after EXCEL 5year results were published, the BBC Newsnight program reported that an independent analysis had yielded an excess risk of $\mathrm{MI}$ with $\mathrm{PCI}$ using this criterion. ${ }^{27}$ It is likely that reporting MIs using this criterion would have overturned the inference of NI which was barely met at 3 years (the upper bound of the difference of $4.0 \%$ was just below the $4.2 \%$ margin). In fact, the fragility index ${ }^{28}$ for NI was 2 , that is, one fewer MI in the CABG arm and one excess MI in the PCI arm would have overturned the NI assessment. ${ }^{29}$

Third, a second BBC Newsnight program reported that the EXCEL report from 2016 only included data censored at 3 years. $^{30}$ An internal memo from the chair of the safety committee advised EXCEL investigators to be completely transparent about reporting all mortality data including those that occurred beyond the censored time. In addition, it is not clear if this review was shared by the members of the 2018 European Society of Cardiology (ESC)/European Association for CardioThoracic Surgery (EACTS) guideline writing committee.

The EXCEL investigators have offered a comprehensive rebuttal to the controversy ${ }^{30}$; but three major cardiac surgical societies have called for an independent review of EXCEL data. EACTS has pulled their support of the ESC revascularization guidelines until the EXCEL trial has been reanalyzed. ${ }^{31}$ The New England Journal of Medicine is also conducting an independent review of the EXCEL trial. ${ }^{32}$

\section{How Should the EXCEL and NOBLE Trials Impact Clinical Practice?}

The current European revascularization guidelines issued in 2018 endorse $\mathrm{PCI}$ as a class IA recommendation (the strongest recommendation) in patients with left main disease with a SYNTAX score of $\leq 22$ (low anatomical complexity), class Ila for patients with a SYNTAX score of 23 to 32 (moderate 


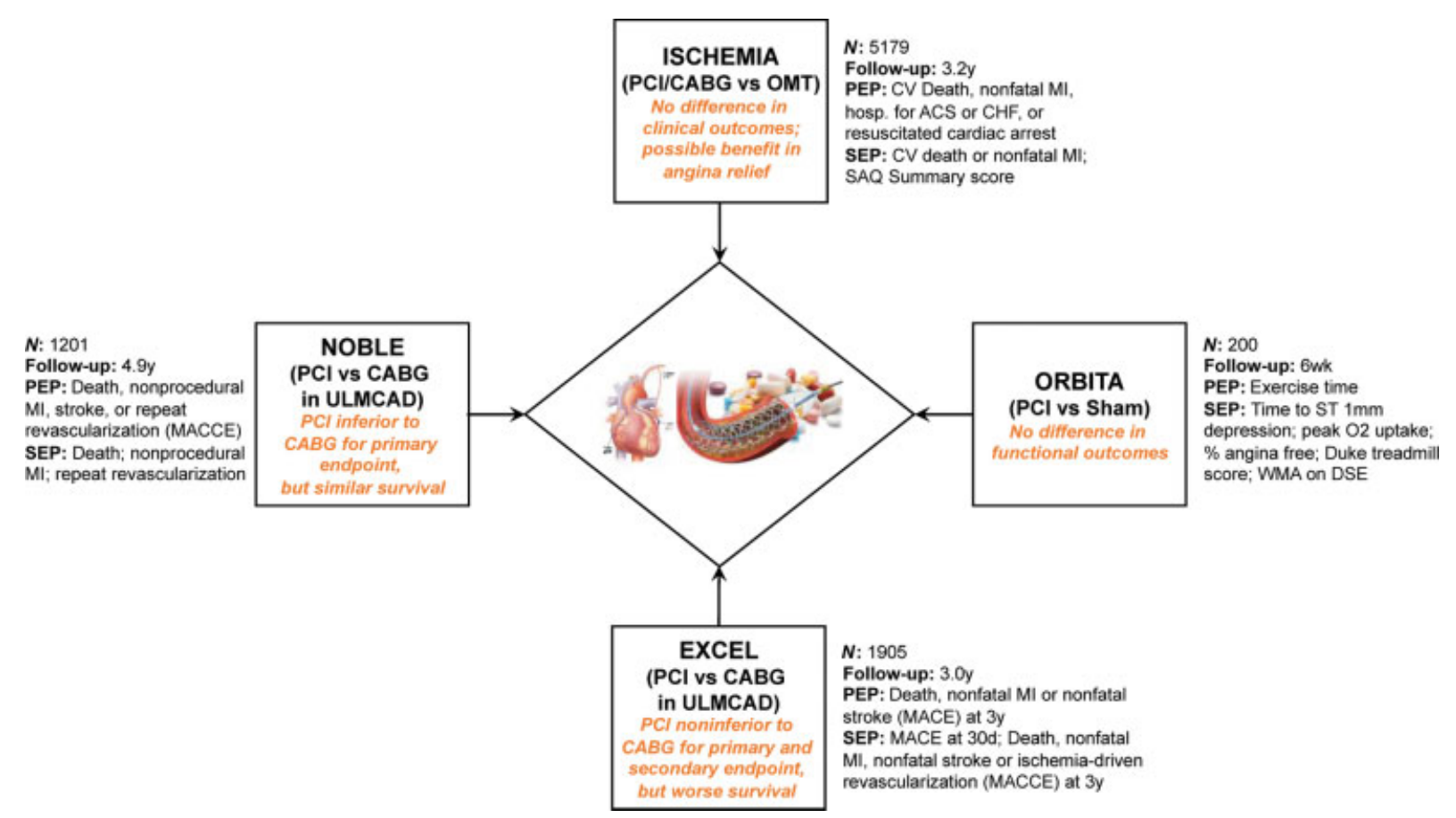

Fig. 1 Summary figure for the four trials. CABG, coronary artery bypass graft; CV, cardiovascular; HR, hazard ratio; MACE, major adverse cardiac events; MI, myocardial infarction; OMT, optimal medical therapy; OR, odds ratio; $\mathrm{PCl}$, percutaneous coronary intervention; PEP, primary efficacy endpoint; SEP, secondary efficacy endpoint.

anatomical complexity), and class IIIB for patients with a SYNTAX score of $>33$ (high anatomical complexity). ${ }^{33}$ The elevation to class IA for low anatomical complexity LMCAD in 2018 guidelines was, in large part, driven by the 3-year results of EXCEL. Considering the totality of the evidence at 5-year follow-up of two large RCTs, and the controversy surrounding the EXCEL trial, we believe that this recommendation should be downgraded. CABG should be the treatment of choice in patients with LMCAD regardless of anatomical complexity. PCI is a reasonable option for those who are at prohibitive risk for surgery or those who prefer not to undergo the more invasive or longer-recovery CABG. The difference in initial morbidity of the two procedures clearly influences patient decision-making. If patients are told the outcomes of PCI and CABG are similar, nearly all will choose $\mathrm{PCI}$. If, however, they are informed that the chance of being alive or having a serious MI are higher with PCI, some would opt for surgery. Decision support tools might be of great help here. ${ }^{34}$

\section{Conclusion}

Recent clinical trials in patients with ischemic heart disease have greatly informed the clinical care of patients with SIHD. ISCHEMIA shows that in the setting of contemporary GDMT, early revascularization in stable patients-even those with moderate to severe ischemia and extensive CAD or advanced CKD-does not prolong life or reduce $\mathrm{MI}$, but might provide relief from angina especially in patients with daily to weekly angina (without advanced CKD). The ORBITA trial for the first time highlights the likelihood of placebo component for angina relief associated with PCI. And in patients with left main coronary disease, the long-term outcomes of EXCEL and NOBLE trials favor CABG over PCI (Summarized in - Fig. 1).

\section{Key Take-Home Messages for the Practicing Clinician from These 5 Trials}

ISCHEMIA/ISCHEMIA-CKD: There is no liability with the initial choice of guideline-directed medical therapy alone over invasive angiography with or without revascularization in patients with angina, stable multivessel CAD, and moderate-to-severe ischemia or advanced CKD.

ORBITA: The advantage of PCI over guideline-directed medical therapy in improving myocardial ischemia is modest at best, and there is a strong placebo component contributing to anginal relief with PCI.

EXCEL/NOBLE: The treatment of choice for patients with unprotected LMCAD of low to moderate anatomical complexity should be CABG because of long-term survival advantage over PCI as well as reductions in spontaneous MI and repeat interventions.

Conflict of Interest

None declared.

\section{References}

1 Maron DJ, Hochman JS, O’Brien SM, et al; ISCHEMIA Trial Research Group. International Study of Comparative Health Effectiveness with Medical and Invasive Approaches (ISCHEMIA) trial: rationale and design. Am Heart J 2018;201:124-135

2 Frye RL, August P, Brooks MM, et al; BARI 2D Study Group. A randomized trial of therapies for type 2 diabetes and coronary artery disease. N Engl J Med 2009;360:2503-2515 
3 Boden WE, O'Rourke RA, Teo KK, et al; COURAGE Trial Research Group. Optimal medical therapy with or without $\mathrm{PCI}$ for stable coronary disease. N Engl J Med 2007;356(15):1503-1516

4 Shaw LJ, Weintraub WS, Maron DJ, et al. Baseline stress myocardial perfusion imaging results and outcomes in patients with stable ischemic heart disease randomized to optimal medical therapy with or without percutaneous coronary intervention. Am Heart J 2012;164(02):243-250

5 Hachamovitch R, Hayes SW, Friedman JD, Cohen I, Berman DS. Comparison of the short-term survival benefit associated with revascularization compared with medical therapy in patients with no prior coronary artery disease undergoing stress myocardial perfusion single photon emission computed tomography. Circulation 2003;107(23):2900-2907

6 Murthy VL, Eagle KA. ISCHEMIA: a search for clarity and why we may not find it. Am Heart J 2018;203:82-84

7 Maron DJ, Hochman JS, Reynolds HR, et al. Initial invasive or conservative strategy for stable coronary disease. N Engl J Med 2020;382:1395-1407

8 Spertus JA, Jones PG, Maron DJ, et al. Health-status outcomes with invasive or conservative care in coronary disease. N Engl J Med 2020;382:1408-1419

9 Bangalore S, Maron DJ, O'Brien SM, et al. Management of coronary disease in patients with advanced kidney disease. N Engl J Med 2020;382:1608-1618

10 Mancini GBJ, Hartigan PM, Shaw LJ, et al. Predicting outcome in the COURAGE trial (Clinical Outcomes Utilizing Revascularization and Aggressive Drug Evaluation): coronary anatomy versus ischemia. JACC Cardiovasc Interv 2014;7(02):195-201

11 Rajkumar CA, Nijjer SS, Cole GD, Al-Lamee R, Francis DP. 'Faith Healing' and 'Subtraction Anxiety' in unblinded trials of procedures: lessons from DEFER and FAME-2 for end points in the ISCHEMIA trial. Circ Cardiovasc Qual Outcomes 2018;11(03): e004665

12 Al-Lamee R, Thompson D, Dehbi H-M, et al; ORBITA investigators. Percutaneous coronary intervention in stable angina (ORBITA): a double-blind, randomised controlled trial. Lancet 2018;391 (10115):31-40

13 Chaitman BR, Skettino SL, Parker JO, et al; MARISA Investigators. Anti-ischemic effects and long-term survival during ranolazine monotherapy in patients with chronic severe angina. J Am Coll Cardiol 2004;43(08):1375-1382

14 Borer JS, Fox K, Jaillon P, Lerebours GIvabradine Investigators Group. Antianginal and antiischemic effects of ivabradine, an I(f) inhibitor, in stable angina: a randomized, double-blind, multicentered, placebo-controlled trial. Circulation 2003;107(06):817-823

15 Francis DP, Al-Lamee R. Percutaneous coronary intervention for stable angina in ORBITA - authors' reply. Lancet 2018;392 (10141):28-30

16 Al-Lamee R, Howard JP, Shun-Shin MJ, et al. Fractional flow reserve and instantaneous wave-free ratio as predictors of the placebo-controlled response to percutaneous coronary intervention in stable single-vessel coronary artery disease. Circulation 2018;138(17):1780-1792

17 Al-Lamee RK, Shun-Shin MJ, Howard JP, et al. Dobutamine stress echocardiography ischemia as a predictor of the placebo-controlled efficacy of percutaneous coronary intervention in stable coronary artery disease: the stress echocardiographystratified analysis of ORBITA. Circulation 2019;140(24): 1971-1980

18 A Placebo-controlled Trial of Percutaneous Coronary Intervention for the Relief of Stable Angina - Full Text View - ClinicalTrials.gov. Available at: https://clinicaltrials.gov/ct2/show/NCT03742050. Accessed August 10, 2020

192012 ACCF/AHA/ACP/AATS/PCNA/SCAI/STS Guideline for the Diagnosis and Management of Patients with Stable Ischemic Heart Disease | Circulation 2012;126:e354-e471

20 Stone GW, Sabik JF, Serruys PW, et al; EXCEL Trial Investigators. Everolimus-eluting stents or bypass surgery for left main coronary artery disease. N Engl J Med 2016;375(23):2223-2235

21 Stone GW, Kappetein AP, Sabik JF, et al; EXCEL Trial Investigators. Five-year outcomes after PCI or CABG for left main coronary disease. N Engl J Med 2019;381(19):1820-1830

22 Holm NR, Mäkikallio T, Lindsay MM, et al; NOBLE investigators. Percutaneous coronary angioplasty versus coronary artery bypass grafting in the treatment of unprotected left main stenosis: updated 5-year outcomes from the randomised, non-inferiority NOBLE trial. Lancet 2020;395(10219):191-199

23 Moussa ID, Klein LW, Shah B, et al. Consideration of a new definition of clinically relevant myocardial infarction after coronary revascularization: an expert consensus document from the Society for Cardiovascular Angiography and Interventions (SCAI). J Am Coll Cardiol 2013;62(17):1563-1570

24 Ruel M, Falk V, Farkouh ME, et al. Myocardial revascularization trials. Circulation 2018;138(25):2943-2951

25 Cho MS, Ahn J-M, Lee C-H, et al. Differential rates and clinical significance of periprocedural myocardial infarction after stenting or bypass surgery for multivessel coronary disease according to various definitions. JACC Cardiovasc Interv 2017;10(15):1498-1507

26 Serruys PW, Morice M-C, Kappetein AP, et al; SYNTAX Investigators. Percutaneous coronary intervention versus coronary-artery bypass grafting for severe coronary artery disease. N Engl J Med 2009;360 (10):961-972

27 Brown DC, Brown E. Surgeons withdraw support for heart disease advice. BBC News2019

28 Khan MS, Ochani RK, Shaikh A, et al. Fragility index in cardiovascular randomized controlled trials. Circ Cardiovasc Qual Outcomes 2019;12:e005755

29 Should Percutaneous Coronary Intervention Be Considered for Left Main Coronary Artery Disease? Insights From a Bayesian Reanalysis of the EXCEL Trial | Cardiology | JAMA Internal Medicine | JAMA Network 2020;180(7):1002-1003

30 EXCEL Leaders Answer Back After BBC Exposé. Medscape

31 EACTSJM A. EACTS responds to BBC Newsnight's investigation on the EXCEL trial. EACTS2019

32 Cohen D, Brown E. New England Journal of Medicine reviews controversial stent study. BMJ British Medical Journal Publishing Group; 2020:368

33 Baumgartner H, Falk V, Bax JJ, et al; ESC Scientific Document Group. 2017 ESC/EACTS Guidelines for the management of valvular heart disease. Eur Heart J 2017;38(36):2739-2791

34 Stacey D, Légaré F, Col NF, et al. Decision aids for people facing health treatment or screening decisions. Cochrane Database Syst Rev 2014;(01):CD001431 